\title{
In vitro antimicrobials activity against endemic Acinetobacter baumannii multiresistant clones
}

\author{
Carlos Hernan Rodriguez ${ }^{1}$, Alejandra De Ambrosio², Milena Bajuk², Mariela Spinozzi ${ }^{2}$, Marcela \\ Nastro ${ }^{1}$, Karina Bombicino ${ }^{1}$, Marcela Radice ${ }^{3}$, Gabriel Gutkind ${ }^{3}$, Carlos Vay ${ }^{1}$, Angela Famiglietti ${ }^{1}$ \\ ${ }^{1}$ Laboratorio de Bacteriología, Departamento de Bioquímica Clínica, Hospital de Clínicas José de San Martín, Facultad de \\ Farmacia y Bioquímica. Universidad de Buenos Aires. Buenos Aires, Argentina \\ ${ }^{2}$ Carrera de Especialistas Bacteriología Clínica Facultad de Farmacia y Bioquímica. Universidad de Buenos Aires. Buenos \\ Aires, Argentina \\ ${ }^{3}$ Cátedra de Microbiología Facultad de Farmacia y Bioquímica. Universidad de Buenos Aires. Buenos Aires, Argentina
}

\begin{abstract}
Background: Multidrug-resistant strains of Acinetobacter baumannii have been reported increasingly around the world. The administration of an association of antibiotics has been proposed to create an active combination and to prevent the emergence of resistance.

Methodology: The activity of colistin, rifampicin, gentamicin, imipenem and their associations was evaluated by means of killing curves in fourteen isolates belonging to three endemic PFGE types, in a university hospital of Buenos Aires city. The 14 isolates were selected on the basis of different mechanisms responsible for resistance to carbapenems and different susceptibility to colistin.

Results: The mechanism responsible for the resistance to imipenem was the production of OXA-23 and OXA-58 carbapenemases. Heteroresistance to colistin was observed in six isolates. The associations colistin-rifampicin and colistin-imipenem were synergistic in heteroresistant isolates and prevented the development of colistin-resistant mutants. The association imipenem-gentamicin was bactericidal in gentamicin susceptible isolates, whereas the association imipenem-rifampicin was always indifferent.

Conclusion: The antimicrobial activity and the presence of synergy are related to the antimicrobials' susceptibilities irrespective of the PFGE type or the OXA-carbapenemase produced.
\end{abstract}

Key words: A. baumannii, time-kill assay, colistin-heteroresistance, rifampicin, imipenem

J Infect Dev Ctries 2010; 4(3):164-167.

(Received 25 October 2009 - Accepted 24 January 2010)

Copyright $\odot 2010$ Rodriguez et al. This is an open-access article distributed under the Creative Commons Attribution License, which permits unrestricted use, distribution, and reproduction in any medium, provided the original work is properly cited.

\section{Introduction}

Acinetobacter baumannii (A. baumannii) is an important opportunistic pathogen, particularly for patients admitted to intensive care units (ICUs); hospital outbreaks caused by this organism have increased worldwide [1-2]. The emergence of $A$. baumannii clinical isolates with resistance to multiple antimicrobials, including broad-spectrum beta (ß)lactams, carbapenems, aminoglycosides, and fluroquinolones was observed. Carbapenem resistance has been correlated mainly with the acquisition of carbapenem hydrolysing class $D \beta$ lactamases [1]. Heteroresistance to colistin has been recently described by Li et al. [3].

In Argentinian hospitals, the dissemination of strains which showed epidemic behaviour and were producers of OXA-23 and OXA-58 carbapenemases has been previously documented [4-5]. For the last 15 years, our hospital has suffered a sustained endemia of multiresistant A. baumannii infections. The pathogen became carbapenem resistant in 1995 and since then, most of the strains have showed susceptibility to colistin and minocycline [6]. Management of A. baumannii multiresistant infection is a medical concern because of the limited therapeutic options available. The administration of an association of antibiotics has been proposed to create an active combination and to prevent the emergence of resistance [1].

The aim of the present study was to investigate in a university hospital of Buenos Aires city, Argentina, the activity of imipenem (IMI), colistin (COL), rifampicin (RIF) and gentamicin (GEN) alone and in double combinations against fourteen multidrug resistant $A$. baumannii isolates, producers of OXA-23 and OXA-58 carbapenemases with a high percentage of colistin heteroresistance. 


\section{Materials and methods}

Fourteen clinical isolates of multidrug-resistant (MDR) A. baumannii were collected from routine clinical samples of patients admitted to the intensive care unit (ICU) during the period June to December 2004. Strains were identified by phenotypic tests and genomic species was determined by Amplified ribosomal DNA restriction analysis (ARDRA) [7]. Pulsed-field electrophoresis (PFGE) was performed with ApaI as described previously [8]. Minimal inhibitory concentrations (MICs) to COL (against the resistant subpopulations and the original strains), RIF, GEN and IMI were performed by agar dilution method and interpreted according to the Clinical and Laboratory Standards Institute (CLSI) [9] and Societe Française de Microbiologie for colistin and rifampicin [10]. Antibiotic powders were obtained from their respective manufacturers (COL, RIF, GEN: Sigma Chemical Co, USA; and IMI: Merck, Sharp \& Dohme, Argentina). The activities of colistin, rifampicin, gentamicin, imipenem and their associations were evaluated by killing curves. The following concentrations were used: colistin $2 \mathrm{mg} /$ liter; rifampicin $4 \mathrm{mg} /$ liter; imipenem $8 \mathrm{mg} /$ liter; gentamicin $4 \mathrm{mg} /$ liter. Briefly, tubes containing cation-supplemented Mueller-Hinton broth (Lab. Britania) with and without antibiotic were inoculated with A. baumannii strains in a log-phase inoculum of roughly $5 \times 10^{5} \mathrm{CFU} / \mathrm{ml}$. Tubes were incubated in an ambient atmosphere at $35^{\circ} \mathrm{C}$. At time 0,4 and 24 hours after inoculation, serial 10 -fold dilutions were performed and aliquots were plated onto nutrient agar. The time-kill studies were performed twice and results were analyzed by using mean colony count values from the duplicate plates for each isolate. The bactericidal activity of single antibiotics or combinations was defined as $\mathrm{a} \geq 3-\log _{10} \mathrm{CFU} / \mathrm{ml}$ decrease in the viable count compared with the initial inoculum. Synergism and antagonism were respectively defined as $\geq 2-\log _{10} \mathrm{CFU} / \mathrm{ml}$ decrease or increase in the viable count with the combination compared with the most active agent alone at different time points. The presence of OXA carbapenemases was searched in the fourteen selected strains by PCR assays using previously described primer pairs for the $b a_{\text {oxa } 23,}$, bla $_{\text {oxa58, }}$, bla $_{\text {oxa } 40}$

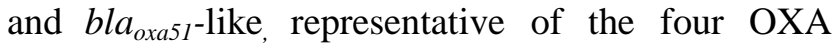
clusters [11]. Phenotypic screening test for metallo- $B$ lactamase production using sodium mercaptoacetic acid disks was also conducted [12].

\section{Results}

Three different PFGE types were delineated in the 14 A. baumannii isolates. These PFGE types were identified as I, III, and IV, maintaining a previous nomenclature [4].

Twelve isolates were resistant to imipenem and only one to colistin. Rifampicin showed a high level of resistance in the majority of the isolates belonging to PFGE type I and a low level in PFGE types III and IV. All isolates belonging to PFGE type III and only one belonging to PFGE type IV were susceptible to gentamicin (Table 1). None of the isolates showed synergy in presence of sodium mercaptoacetic acid; thus they were presumed not to possess metallo- $\beta$ lactamases [12].

In respect to the PCR results for the

Table 1. Pharmacodynamic effects of imipenem, rifampicin and gentamicin against A. baumannii endemic PFGE type producers of OXA-23 and OXA-58.

\begin{tabular}{|c|c|c|c|c|c|c|c|c|c|c|c|c|c|c|c|}
\hline \multirow{4}{*}{$\begin{array}{l}\text { PFGE } \\
\text { types }\end{array}$} & \multirow[t]{4}{*}{$\mathrm{N}$} & \multicolumn{3}{|c|}{ MIC } & \multirow[t]{4}{*}{ CHDL } & & & \multirow{2}{*}{\multicolumn{2}{|c|}{ changes }} & \multirow{2}{*}{\multicolumn{2}{|c|}{$\begin{array}{l}\text { referring to } \\
\text { in } \log _{10}\end{array}$}} & \multirow{2}{*}{\multicolumn{2}{|c|}{$\begin{array}{l}\text { the original } \\
\mathrm{CFU} / \mathrm{ml}\end{array}$}} & \multicolumn{2}{|c|}{ inoculum } \\
\hline & & & \multicolumn{2}{|c|}{$(\mu \mathrm{g} / \mathrm{ml})$} & & \multirow{2}{*}{\multicolumn{2}{|c|}{ IMI }} & & & & & & & & \\
\hline & & \multirow[t]{2}{*}{ IMI } & RIF & GEN & & & & \multicolumn{2}{|c|}{ RIF } & \multicolumn{2}{|c|}{ GEN } & \multicolumn{2}{|c|}{ IMI-RIF } & \multicolumn{2}{|c|}{ IMI-GEN } \\
\hline & & & & & & 4 hs. & $24 \mathrm{hs}$. & $4 \mathrm{hs}$. & $24 \mathrm{hs}$. & $4 \mathrm{hs}$ & $24 \mathrm{hs}$ & $4 \mathrm{hs}$ & $24 \mathrm{hs}$ & $4 \mathrm{hs}$ & $24 \mathrm{hs}$ \\
\hline I & 6 & 32 & $>128$ & 16 & 23 & $+1,2$ & $+2,1$ & $+1,4$ & $+2,4$ & $+1,0$ & $+3,5$ & $+1,2$ & $+2,5$ & $+0,5$ & $+3,1$ \\
\hline I & 1 & 0,5 & 2 & $>16$ & & -2 & $-3,5$ & $-2,0$ & $-3,0$ & \multicolumn{2}{|c|}{ NA } & $-2,9$ & $-3,8$ & & \\
\hline III & 3 & 32 & 8 & 0,5 & 58 & $+1,1$ & $+2,0$ & $+2,0$ & $+2,1$ & $-1,0$ & $-2,3$ & $+1,0$ & $+1,1$ & $-1,0$ & $-3,1$ \\
\hline IV & 2 & 32 & 8 & $>16$ & 23 & 0 & $+2,0$ & $+1,1$ & $+1,1$ & $-0,7$ & $-1,0$ & $+1,1$ & 0 & $-0,7$ & $-1,8$ \\
\hline IV & 1 & 64 & 8 & 2 & 23 & 0 & $+1,6$ & 0 & $-1,1$ & $-1,0$ & $-1,9$ & $+1,2$ & $-0,1$ & $-1,0$ & $-4,0$ \\
\hline IV & 1 & 1 & 2 & $>16$ & & $-1,0$ & $-4,0$ & $-1,0$ & $-3,9$ & & & & & $-1,0$ & $-3,9$ \\
\hline
\end{tabular}


carbapenemases, the bla oxa 51-like ß-lactamase gene was detected in all fourteen strains whereas $b l a_{\text {oxa2 } 3}$ was detected in carbapenem-resistant isolates in PFGE type I and IV and bla oxass in PFGE type III (Table 1).

Heteroresistance to colistin was observed in six isolates (Table 2).
III and in one isolate in PFGE type IV if they were susceptible to GEN and showed synergy when combined with IMI (Table 1). The GEN concentration used is the one achieved in serum and lung tissue; hence this antimicrobial association could be a possible therapeutic option for the treatment of these MDR isolates.

Table 2. Pharmacodynamic effects of colistin and colistin plus rifampicin or imipenem against A. baumannii resistant, heteroresistant or non-heteroresistant isolates.

\begin{tabular}{|c|c|c|c|c|c|c|c|c|c|c|}
\hline \multirow[t]{3}{*}{$\begin{array}{l}\text { PFGE } \\
\text { types }\end{array}$} & \multirow[t]{3}{*}{$\mathrm{N}$} & \multirow[t]{3}{*}{ CHDL } & \multirow[t]{3}{*}{ S. $\operatorname{col}^{1}$} & \multirow[t]{3}{*}{$\begin{array}{r}\text { MIC COL } \\
(\mu \mathrm{g} / \mathrm{ml})\end{array}$} & \multicolumn{2}{|c|}{ Average changes } & \multicolumn{2}{|c|}{$\frac{\text { referring to the original }}{\left(\log _{10} \mathrm{CFU} / \mathrm{ml}\right)}$} & \multicolumn{2}{|c|}{ inoculum } \\
\hline & & & & & \multicolumn{2}{|c|}{$\mathrm{COL}$} & \multicolumn{2}{|c|}{ COL-RIF } & \multicolumn{2}{|c|}{ COL-IMI } \\
\hline & & & & & $4 \mathrm{hs}$ & $24 \mathrm{hs}$ & 4hs & $24 \mathrm{hs}$ & $4 \mathrm{hs}$ & $24 \mathrm{hs}$ \\
\hline \multirow[t]{3}{*}{ I } & 3 & 23 & $\mathrm{H}^{2}$ & 1 & $-0,5$ & $-3,5$ & $-4,0$ & $-4,0$ & -4 & $-3,9$ \\
\hline & 3 & $23 *$ & no $\mathrm{H}^{3}$ & 1 & $-2,9$ & $-3,9$ & $-3,0$ & $-3,9$ & -4 & $-3,9$ \\
\hline & 1 & 23 & $\mathrm{R}^{4}$ & 32 & $-1,8$ & $-1,5$ & $+1,2$ & $-2,9$ & $-2,2$ & $-3,0$ \\
\hline III & 3 & 58 & $\mathrm{H}$ & 1 & $-1,8$ & $+1,1$ & $-2,0$ & $-3,9$ & $-1,9$ & $-4,0$ \\
\hline IV & 4 & $23 * *$ & no $\mathrm{H}$ & 1 & $-3,9$ & $-4,1$ & $-3,8$ & $-4,0$ & $-3,9$ & $-3,9$ \\
\hline
\end{tabular}

1: Susceptibilities of colistin; 2: Heteroresistant isolates; 3 : non-heteroresistant isolates; 4 : resistant isolates, N: Number of isolates studied. CHDL: Carbapenem-hydrolysing Class D oxacillinase, ${ }^{*}$ only in two strains, $* *$ in three strains.

\section{Discussion}

In the present study the concentrations for the time-kill experiments were selected according to the serum-levels and the breakpoints established by CLSI, because we believe that these concentrations show better correlations with therapeutic conditions than MIC multiples in MDR strains [13-14].

In time-kill studies, imipenem showed bactericidal activity against only two carbapenemsusceptible strains, confirming its excellent activity. We observed bacteriostatic activity at four hours in all the IMI resistant strains irrespective of PFGE type or production of acquired Class D carbapenemases (Table 1). Similar results were reported by Tripodi et al. but we believe that this behaviour would not have clinical relevance and that it would be related to the heteroresistance to carbapenems [15].

Colistin sulphate showed bactericidal activity before $4 \mathrm{hs}$ of incubation in non-heteroresistant isolates. In heteroresistant isolates we observed a decrease in the bactericidal activity or regrowth of resistant sub-populations (MIC 4-fold higher than in original strains) at 24 hours of incubation. Bacteriostatic activity was detected in the resistant isolate (Table 2). We demonstrated bactericidal activity with GEN in isolates belonging to PFGE type
In A. baumannii infections, RIF has been used in combinations with other antimicrobials to prevent the emergence of resistant mutants. Three rifampicin MIC values were obtained: 2, 8, and $>128 \mathrm{ug} / \mathrm{ml}$. Bactericidal activity was detected only in the isolate with an MIC of $2 \mathrm{ug} / \mathrm{ml}$. The association IMI-RIF did not show ability to achieve synergy in isolates harbouring OXA-23 or OXA-58, because the RIF concentrations used were always lower than the MIC value (sub-MIC) (Table 1). Tripodi et al. reported synergy with the combination IMI-RIF against producers of OXA-58 isolates using RIF concentrations higher than the MIC value in killing curves [15]. However, we observed that RIF plus COL showed synergy by inhibition of resistant subpopulations in heteroresistant isolates irrespective of the RIF MIC values and we detected synergy at 24 hours in the isolate resistant to COL despite its high MIC to both antimicrobials (Table 2). Similarly, the combination COL plus RIF was constantly synergistic against $S$. marcescens, an organism that is intrinsically resistant to polimixyns [16].

In non heteroresistant isolates we were unable to demonstrate synergy between COL and IMI in isolates harbouring OXA-23 or OXA-58, partly because COL alone showed a rapid initial bactericidal effect (before four hours of incubation). 
As it was previously mentioned, in heteroresistant isolates both COL-RIF and COL-IMI combinations were synergistic in inhibition of regrowth (Table 2). Similar findings were obtained with the colistinminocycline associations [17]. Few synergy studies have been conducted in A. baumannii COL resistant strains. In agreement with a previous report by Yoon et al. [13], we detected bactericidal activity between IMI-COL or COL-RIF at 4 and 24 hours, respectively (Table 2). The rapid permeabilization of the outer membrane would enhance the action of the other antimicrobials in COL susceptible strains, whereas the synergistic mechanism in COL resistant isolates remains unknown. In accordance with Principe et al., we observed different intraclonal synergistic activities that reflect the complex interactions of multiple mechanisms of antimicrobial resistance in $A$. baumannii [18].

In conclusion, these studied strains belong to the three major endemic PFGE types in Buenos Aires city [4]. Thus, our results could be applied in our hospital and other medical centers for the treatment of multiresistant A. baumannii infections. From our data, we showed that the combination of COL plus RIF or IMI was effective (always bactericidal and synergistic against heteroresistant isolates) and it prevented the selection of resistance. In this study we demonstrated that the antimicrobial activity and the presence of synergy are related to the antimicrobials' susceptibilities irrespective of the PFGE types or the OXA-carbapenemase produced.

\section{References}

1. Peleg AY, Seifert H, Paterson D (2008) Acinetobacter baumannii: Emergence of successful pathogen. Clin Microbiol Rev 21: 538-92.

2. Zarrilli R, Giannouli M, Tomasone F, Triassi M, Tsakris A (2009) Carbapenem resistance in Acinetobacter baumannii: the molecular epidemic features of an emerging problem in health care facilities. J Infect Dev Ctries 3: 335-41.

3. Li J, Rayner CR, Nation RL, et al. (2006) Heteroresistance to colistin in multidrug-resistant Acinetobacter baumannii Antimicrob Agents Chemother 50: 2946-50.

4. Barbolla RE, Centron D, Di Martino A, et al. (2003) Identification of an epidemic carbapenem-resistant Acinetobacter baumannii strain at hospitals in Buenos Aires city. Diag Microbiol Inf Dis 45: 261-64.

5. Merkier A, Catalano M, Ramirez M, Quiroga C, Orman B, Ratier L, Famiglietti A., Vay C, Di Martino A, Kaufmann S, Centron D (2008) Polyclonal spread of bla $\mathrm{bxa}_{\text {-58 }}$ in Acinetobacter baumannnii isolates from Argentina. J Infect Dev Ctries 2: 235-40.

6. Rodríguez CH, Juárez J, de Mier C, et al. (2003) Resistencia a antibióticos de bacilos gram negativos aislados en unidades de cuidados intensivos. Análisis comparativo de dos años (1998-2001). Medicina (Buenos Aires) 63: 21-27.
7. Vaneechoutte M, Dijkshoorn L, Tjernberg I, Elaichouni A, de Vos P, Claeys G, et al. (1995) Identification of Acinetobacter genomic species by amplified ribosomal DNA restriction analysis. J Clin Microbiol 33:11-15.

8. Seirfert H, Dolzani L, Bressan R et al. (2005) Standardization and interlaboratory reproducibility assessment pulsed-field electrophoresis generated fingerprints of Acinetobacter baumannii. J Clin Microbiol 43: 4328-35.

9. Clinical and Laboratory Standards Institute. (2006) Methods for dilution antimicrobial susceptibility test for bacteria that grow aerobically. Approved standard M7-A7. Clinical and Laboratory Standards Institute, Wayne PA.

10. SFM Antibiogram Committee (2003) Comite de l'Antibiogramme de la Société Française de Microbiologie report. Int J Antimicrob Agents. 21: 364-91.

11. Woodford N, Ellington M, Coehlo J, Turton J, Ward M, Brown S, Amyes S, Livermore D (2006) Multiplex PCR for genes encoding prevalent OXA carbapenemases in Acinetobacter spp. Int J Antimicrob Agents 27: 351-53.

12. Lee K, Lim S, Yum JH, Yong D, Chong Y (2003) Evaluation of the Hodge test and the imipenem-EDTA double-disk synergy test for differanting metallo-blactamase-producing isolates of Pseudomonas spp. and Acinetobacter spp. J Clin Microbiol 41: 4623-29.

13. Yoon J, Urban C, Terzian C, Mariano N, Rahan J (2004) In vitro double and triple synergistic activities of polymyxin $\mathrm{B}$, imipenem, and rifampin against multidugr-resistant Acinetobacter baumannii. Antimicrob Agents Chemother 48: 753-57.

14. Montero A, Ariza J, Corbella X, Doménech A, Cabellos C, Ayats J, Tubau F, Borraz C, Gudiol . (2004) Antibiotic combinations for serious infections caused by carbapenemresistant Acinetobacter baumannii in a mouse pneumonia model. J Antimicrob Chemother 54: 1085-91.

15. Tripodi MF, Durante-Magnoni E, Fortunato R, Utili R, Zarrilli R (2007) Comparative activities of colistin, rifampicin, imipenem and sulbactam-ampicillin alone or in combination against epidemic multidrug-resistant Acinetobacter baumannii isolates producing OXA-58 carbapenemases. Int J Antimicrob Agents 30: 537-40.

16. Landman D, Georgescu C, Martin A, Quale J (2008) Polymyxins revisited. Clin Microbiol Rev 21: 449-65.

17. Tan $\mathrm{T}, \mathrm{Ng} \mathrm{L}$, Tan E, Huang G (2007) In vitro effect of minocycline and colistin combinations on imipenemresistant Acinetobacter baumannii clinical isolates. J Antimicrob Chemother 60: 421-23.

18. Principe L, D’Arezzo S, Capone A, Petrosillo N, Visca P (2009) In vitro activity of tigecycline in combination with various antimicrobials against multidrug resistant Acinetobacter baumannii. Ann Clin Microbiol Antimicrob 8: 18.

\section{Corresponding author}

Rodriguez Carlos Hernan

Departamento de Bioquímica Clínica

Hospital de Clínicas José de San Martín

Córdoba 2351 [1120] Buenos Aires. Argentina

Telephone: [54-11]45028597 Fax: [54-11]5950-8694

Email: Carlos_HernanRodriguez@hotmail.com.ar

Conflict of interests: No conflict of interests is declared. 